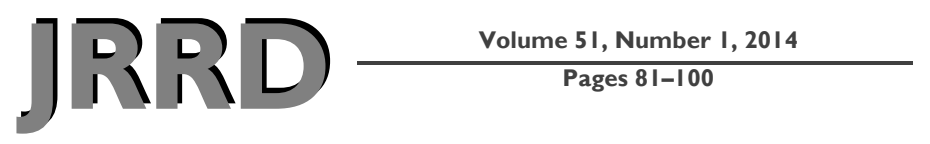

\title{
Proposed comprehensive ototoxicity monitoring program for VA healthcare (COMP-VA)
}

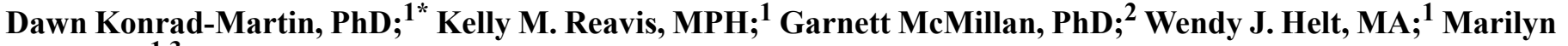 \\ Dille, $\mathbf{P h D}^{\mathbf{1 , 3}}$ \\ ${ }^{1}$ National Center for Rehabilitative Auditory Research, Portland Department of Veterans Affairs Medical Center, Port- \\ land, OR; Departments of ${ }^{2}$ Public Health and Preventive Medicine and ${ }^{3}$ Otolaryngology/HNS, Oregon Health \& Sci- \\ ence University, Portland, OR
}

\begin{abstract}
Prevention and rehabilitation of hearing loss and tinnitus, the two most commonly awarded service-connected disabilities, are high priority initiatives in the Department of Veterans Affairs (VA). At least 4,000 Veterans, most with significant hearing loss, will receive cisplatin this year, with more than half sustaining permanent hearing shift and nearly $40 \%$ developing new tinnitus. With improved survivability following cancer treatment, Veterans treated with cisplatin are approached with the dual goals of effective treatment and preserved quality of life. This article describes COMP-VA, a comprehensive ototoxicity monitoring program developed for VA patients receiving cisplatin. The program includes an individualized pretreatment prediction model that identifies the likelihood of hearing shift given cisplatin dose and patient factors. It supports both manual and automated hearing testing with a newly developed portable audiometer capable of performing the recommended procedures on the chemotherapy unit during treatment. It also includes objective methods for identifying outer hair cell changes and predicting audiogram changes using distortion-product otoacoustic emissions. We describe this program of evidence-based ototoxicity monitoring protocols using a case example to give the reader an understanding of how this program would be applied, along with a plan for future work to accomplish the final stages of program development.
\end{abstract}

Key words: aural rehabilitation, chemotherapy, cisplatin, COMP-VA, distortion-product otoacoustic emissions, DPOAE, hearing, OtoID, ototoxicity monitoring, sensitive range for ototoxicity.

\section{INTRODUCTION}

Prevention and rehabilitation of hearing loss and tinnitus are high priority initiatives in the Department of Veterans Affairs (VA). In the last decade, more than 33,000 Veterans nationally were treated with cisplatin chemotherapy at VA medical centers (VAMCs) (VA Informatics and Computing Infrastructure database, accessed

Abbreviations: AAA $=$ American Academy of Audiology, ANSI $=$ American National Standards Institute, ASHA = American Speech-Language-Hearing Association, $\mathrm{AUC}=$ area under the Receiver Operating Characteristic curve, COMP-VA = comprehensive ototoxicity monitoring program for Department of Veterans Affairs, DPOAE = distortion-product otoacoustic emission, HIPAA $=$ Health Insurance Portability and Accountability Act of 1996, I/O = input-output, MPANL = maximum permissible ambient noise level, NCRAR $=$ National Center for Rehabilitative Auditory Research, RR\&D = Rehabilitation Research and Development, SPL $=$ sound pressure level, $\mathrm{SRO}=$ sensitive range for ototoxicity, $\mathrm{TOMI}=$ Tinnitus Ototoxicity Monitoring Interview, VA = Department of Veterans Affairs, VAMC = VA medical center.

*Address all correspondence to Dawn Konrad-Martin, PhD; Portland VA Medical Center, National Center for Rehabilitative Auditory Research, 3710 SW US Veterans Hosp Rd, P5-NCRAR, Portland, OR 97239; 503-220-8262, ext 52962; fax: 503-721-1402. Email: dawn.martin@va.gov http://dx.doi.org/10.1682/JRRD.2013.04.0092 
2012 May). Cisplatin is an antineoplastic agent used for the treatment of a variety of adult cancers, including bladder, testicular, gynecologic, head and neck, and lung. If trends continue, more than 4,000 Veterans will receive cisplatin this year, with more than 50 percent sustaining a permanent hearing shift and 39 percent developing new tinnitus [1-3]. Importantly, in our experience, the average age of Veterans entering treatment is $60 \mathrm{yr}$ and most report a history of significant noise exposure [4-5], which accounts for the high rate of preexisting hearing loss in this population. It is well documented that hearing loss adversely impacts quality of life [6]; psychosocial functioning [7]; and one's ability to obtain, process, and understand basic health information [8]; therefore, it is an undeniably important side effect to monitor during chemotherapy. Furthermore, health-related quality of life concerns are important to oncologists because improved treatments and early diagnoses have increased long-term cancer survivability. The approach of VA is to provide cancer management that is patient centered and directed toward improving the quality of life following treatment.

Within the inner ear, cisplatin primarily damages the cochlea, resulting in permanent, usually bilateral, highfrequency sensorineural hearing loss in approximately 60 percent of adults treated with the drug [9]. This damage occurs in a relatively orderly manner of base (high frequency) to apex (low frequency) destruction [10] primarily of the outer hair cells, which are sensory receptor cells that provide for the large dynamic range and exacting frequency selectivity that characterizes human hearing. Damage also occurs to the marginal cells of the stria vascularis [11-14], which provides the electrochemical drive for the outer hair cells and spiral ganglion cells of the auditory nerve. A main mechanism of cisplatin ototoxicity is associated with the production of free radicals [15]. Overabundance of these unstable molecules overloads intracellular antioxidant enzymes and leads to a cascade of damaging oxidative reactions and upregulation of programmed cell death. Depleting the natural defense system of cells leaves them especially susceptible to further ototoxic damage with each additional treatment. The resultant peripheral hearing loss from this cell damage has been shown to be dose dependent in both animals [11] and humans [16].

Currently, only a small fraction of Veterans undergoing chemotherapy with cisplatin are systematically monitored for signs of ototoxicity. Too often, Veterans in treatment are asked to arrange for audiological testing once debilitating hearing loss is already apparent to the patient or treatment team. This testing must then be coordinated with a patient's already overburdened treatment schedule and into audiology clinic time slots that are routinely scheduled months in advance. Further, these appointments include lengthy diagnostic testing when a narrowly focused hearing screening done at each cisplatin-treatment visit would seem to be a better approach.

Nearly $30 \mathrm{yr}$ of prospective ototoxicity monitoring and research by the VA Rehabilitation Research and Development (RR\&D) National Center for Rehabilitative Auditory Research (NCRAR) have shown that education and frequent communication with the monitoring audiologist improve the likelihood that oncologists will seek out and use information about ototoxic hearing changes for purposes of fine-tuning chemotherapy, when medically appropriate, to avoid disabling hearing loss. It has also become clear that audiology equipment and staffing limitations need to be overcome in order to consistently identify those patients who face the greatest risk for preventable hearing loss and to support those who need extra help and motivation to access VA's comprehensive range of hearing loss and tinnitus rehabilitation services. Further, the VA's existing mechanisms for tracking patients throughout the system need to be exploited in order to ensure patients receive the audiological services they may need at various stages of cancer treatment and survivorship.

The purpose of this article is to report on a complete program of evidence-based ototoxicity monitoring protocols, the comprehensive ototoxicity monitoring program for VA (COMP-VA), developed at the NCRAR. COMPVA was designed to achieve a range of customizable clinical objectives and to test all patients during treatment regardless of how sick they are or their ability to reliably take a hearing test using conventional methods. This program includes an individualized pretreatment prediction model that identifies the likelihood of hearing shift from cisplatin and supports both manual and automated hearing testing with a newly developed portable audiometer capable of performing the recommended procedures on the chemotherapy unit during treatment. Also included are two objective test methods for identifying outer hair cell changes and predicting audiogram changes using distortion-product otoacoustic emissions (DPOAEs). The primary goal of COMP-VA is early detection of ototoxicity in order to optimize both audiological outcomes and therapeutic management of patients receiving cisplatin 
chemotherapy. This goal is to be accomplished using a team-based approach made possible in large part by delivering COMP-VA services chairside on the oncology treatment unit.

\section{METHODS}

\section{Programmatic Research Supporting Proposed Protocols}

\section{Serial Hearing Testing Using OtoID Portable Audiometer}

COMP-VA is designed to be administered on the chemotherapy treatment unit just prior to treatment (chairside). This eliminates the need for patients to make additional trips to the VA and the need for scheduled hearing evaluations in the audiology clinic. To accomplish this, researchers at the NCRAR designed and engineered a portable ototoxicity identification device, the OtoID, that can provide reliable and accurate hearing thresholds on the hospital ward during treatment [17].

The OtoID portable audiometer is comprised of a touch screen computer module, Sennheiser HDA200 circumaural headphones (Sennheiser Electronic Corp; Old Lyme, Connecticut) modified with ambient noise monitoring microphones and custom audiometric testing software. Unlike most commercially available portable audiometers, the OtoID is capable of obtaining air conduction thresholds at $500-20,000 \mathrm{~Hz}$ in $1 / 6$ th octave intervals with a dynamic range of $115 \mathrm{~dB}(-10$ to $105 \mathrm{~dB})$. The OtoID meets or exceeds all American National Standards Institute (ANSI) S3.6-2010 class 4 and high frequency audiometer specifications for reference equivalent threshold sound pressure levels (SPLs), frequency accuracy and purity, attenuator linearity and rise/fall characteristics, and absence of unwanted acoustic signals [18]. A detailed description of the OtoID is provided in Jacobs et al. [18].

The device has both manual (audiologist-directed testing) and automated (patient self-testing) modes. Figure 1 shows the OtoID being used for testing a Veteran in the automated mode. The automated mode prompts the user when headphone placement is incorrect and employs catch trials to achieve bias-free threshold estimates. Puretone threshold results for each patient are stored in the OtoID, which has the capability to recall previous test results via a patient identifier (e.g., a Health Insurance Portability and Accountability Act of 1996 [HIPAA]compliant patient number). Additionally, the OtoID measures the ambient room noise at earphone level before

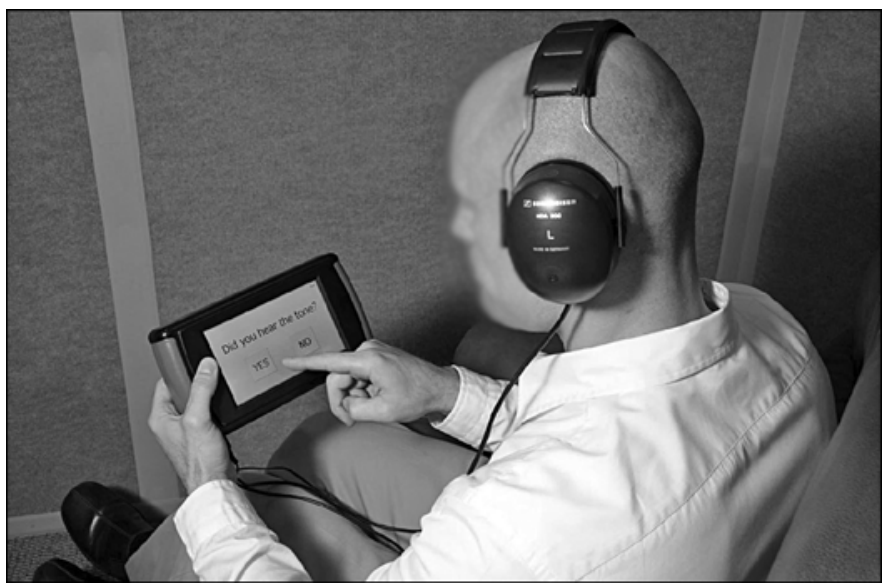

Figure 1.

Veteran using OtolD in automated (self-test) mode. Veteran is alerted to upcoming listening interval. Test tone is either played or catch trial occurs in which no tone is played. Patient is then instructed to respond via touch screen whether or not tone was heard. Earphones shown are Sennheiser HDA 200. Reprinted from Dille et al. [17].

each tone presentation. If the ambient noise exceeds ANSI S3.1-1999 maximum permissible ambient noise levels (MPANLs), the tone is not presented and the patient is instructed to either wait until the room is quiet or move to a quieter location.

Ongoing development of the OtoID includes the secure exchange of information between the device and the VA's computerized patient records system and telehealth capabilities. Sending HIPAA-compliant results from the OtoID built-in cell phone modem to a VA email address via secure text message is nearly complete. Automated testing combined with telehealth capabilities provides major advantages of the OtoID over current modes of ototoxicity monitoring. The automated test mode saves audiological professional time by having the patient conduct the behavioral hearing screening portion of the monitor visit. Once the patient completes self-testing and the results are received in the audiology clinic, the audiologist can then travel to the treatment unit, complete additional tests (otoscopy and tympanometry, screen-fail follow-up testing if necessary), and discuss results with the patient and the oncology team, ensuring that fully informed and timely treatment decisions can be made.

\section{Behavioral Hearing Screening Using COMP-VA}

Ototoxic damage must be detected by assessing auditory function directly. Standard air conduction testing in 
conventional and extended high frequencies is possible using the OtoID. However, the sensitive range for ototoxicity (SRO) technique [19] is also supported by COMPVA. The SRO technique is proposed as the behavioral hearing test method of choice for ototoxicity monitoring, as long as the Veteran is able to provide reliable hearing results despite the exhaustion caused by chemotherapy treatment. The SRO technique was designed to identify hearing shifts within an individualized range of relatively high frequencies so that treatment changes can be considered, if deemed necessary, to prevent hearing loss from spreading to the frequencies considered most important for understanding speech (those up to $4,000 \mathrm{~Hz}$ ).

The SRO is determined based on each patient's preexposure hearing and consists of seven frequencies spaced 1/6-octave apart, spanning 1 octave near a patient's operationally defined high frequency hearing limit. Significant hearing shifts during ototoxicity monitoring have been defined by the American Speech-Language-Hearing Association (ASHA) [20] as a $20 \mathrm{~dB}$ decrease at any one test frequency, a $10 \mathrm{~dB}$ decrease at any two adjacent test frequencies, or the loss of response at any three consecutively tested frequencies where responses were previously obtained. ASHA's criteria for a significant hearing shift when used with the SRO result in a reliable test $[5,18]$. The SRO technique is also sensitive $(94 \%$ detection) regardless of whether the SRO falls above $8,000 \mathrm{~Hz}$ $[19,21]$ or below $8,000 \mathrm{~Hz}$ [4]. The theorized basis for this high sensitivity is that the high frequency-coded base is the most physiologically vulnerable area within the cochlea to cisplatin-related damage, and this damage progresses from base to apex.

Currently, most ototoxicity programs monitor in the conventional frequency range only, even though numerous studies show greater sensitivity is achieved by testing frequencies above $8,000 \mathrm{~Hz}$ [22]. For example, one clinical study showed that if only conventional frequency testing $(\leq 8,000 \mathrm{~Hz})$ were used, 36 percent of initial (early detection) changes would have gone undetected [4], potentially missing the opportunity to prevent changes in the more functionally critical conventional frequencies. Further, use of the SRO screening protocol together with tests to rule out ear disease or obstruction (otoscopy, tympanometry) reduces testing time substantially in comparison to the full diagnostic evaluation that is commonly done, which includes otoscopy, tympanometry, air and bone conduction, and speech testing. Extensive audiometric evaluations, typically done in the audiology clinic, are best saved until the patient has finished treatment and, perhaps, is ready to pursue hearing aid amplification.

We have recently confirmed that the risk for hearing shift from cisplatin is primarily related to the cumulative drug dose and, established in three separate data sets, that patients with better preexposure hearing have a greater risk of hearing change within the SRO at a given dose compared with patients with worse preexposure hearing [1-2,23]. Previous studies that examined extent of preexposure hearing as a potential risk factor for ototoxicity have conflicting results [24-25]. Differences across studies can be understood in terms of the differences in testing methodology, specifically the different test frequency ranges used. Hearing tests lose sensitivity for subjects with good preexposure hearing when testing does not include frequencies near the high frequency hearing limit. The increased vulnerability of the cochlea near its base, i.e., more hearing loss per unit cisplatin dose, was likely masked in previous studies by the decreased sensitivity of conventional frequency testing for subjects with good preexposure hearing.

\section{DPOAE Screening Using COMP-VA}

In their respective clinical guidelines for ototoxicity monitoring, ASHA and the American Academy of Audiology (AAA) have proposed that both behavioral and nonbehavioral (objective) measures of auditory function be routinely used at the baseline evaluation because it is not clear which patients will become unable to provide reliable behavioral thresholds during treatment $[20,26]$. By our estimation, at least 30 percent of patients who receive chemotherapy do, in fact, become unable to respond reliably during testing [27-28]. Using data from our recently completed ototoxicity research project at NCRAR, 50 of the 118 (41\%) study subjects treated with cisplatin became untestable at some point in their treatment due to fatigue, illness, disease progression, or unwillingness to go to a sound suite for testing [2].

DPOAEs are objective measures able to identify cochlear changes associated with ototoxicity [29-30]. DPOAEs depend on the physiological status of the outer hair cells, which are the initial site of ototoxic damage and, therefore, should be sensitive to hearing shifts [31]. DPOAEs are elicited using two tones that are close in frequency and presented simultaneously. Responses are influenced by hearing loss at the DPOAE frequency $\left(2 \mathrm{f}_{1}\right.$ $f_{2}$ ), the eliciting frequencies $\left(f_{1}\right.$ and $f_{2}$, where $\left.f_{1}<f_{2}\right)$, and comparatively higher frequencies coded by more basal 
cochlear regions [29,32-33]. Although current ASHA and AAA guidelines urge that DPOAE testing be included programmatically, little guidance is currently given as to how to interpret the findings. COMP-VA addresses this information gap.

One proposed DPOAE screening protocol is analogous to the behavioral SRO technique in that it includes an initial broad frequency sweep done in 1/6-octave steps to identify the highest frequency at which a valid response of +6 signal-to-noise ratio can be measured followed by additional monitoring of several frequencies near this limit at each chemotherapy treatment. Using a machine learning paradigm, we established that DPOAE shift metrics, obtained by comparing DPOAE baseline results to monitoring results, alone are sensitive predictors of hearing change in the SRO region, but when DPOAEs are combined in a multivariate model with cumulative drug dose and baseline hearing levels, sensitivity increases without causing unacceptably high false positive rates. In the multivariate model, DPOAE testing has proven to be sensitive to early ototoxic hearing shift using either input-output (I/O) intensity functions, i.e., decrease in level while holding frequency constant (area under the Receiver Operating Characteristic curve $[\mathrm{AUC}]=0.90)[22]$, or using fine step frequency measures, i.e., small frequency changes while holding intensity constant (AUC $=0.79)$ [2]. Providing objective techniques for identifying shifts in behavioral pure-tone thresholds when a patient is unable to provide reliable hearing test results, due to extreme illness or fatigue characteristic of chemotherapeutic treatment, is a very important and often overlooked programmatic component.

Another proposed DPOAE protocol involves the use of test-retest reference limits based on normal variability in serial DPOAE level plotted as a function of $\mathrm{f} 2$ frequency levels obtained in a nonexposed (to cisplatin) group of Veteran research participants. DPOAE level shifts obtained during ototoxicity monitoring that are greater than the decibel shift that marks the upper 90th percentile from the reference population identify a clinically significant DPOAE change [34]. Because there is no commonly accepted gold standard of outer hair cell damage in humans, test accuracy can never be completely known for this method. However, a false positive rate of DPOAE change can be estimated for a tester or group of testers using group variability from a nonexposed control group.
Even though many Veterans have preexisting hearing loss, most have DPOAEs that can be monitored for changes. In a prior study in which we examined DPOAEs among individuals with confirmed behavioral hearing shifts following cisplatin administration, most subjects $(82 / 90$ or $91 \%)$ had DPOAEs that were able to be successfully monitored for changes [3]. Importantly, in a separate trial investigating DPOAE test performance for detecting ototoxicity, only 10 percent of ears had to be excluded for a lack of DPOAEs at baseline [2]. Experience has shown that DPOAE measures are maximally sensitive to ototoxicity if they are within one octave of the behaviorally tested SRO [3]. In practice, we have found that there are many instances in which druginduced pure-tone threshold shifts are restricted to a DPOAE frequency range that did not have a valid response at the baseline test session. In such cases, we have found that DPOAEs obtained at comparatively lower $\mathrm{f}_{2}$ frequencies are still useful for predicting hearing shifts, either because DPOAEs are sensitive to preclinical damage or are influenced by threshold shifts at comparatively higher frequencies than the eliciting primaries [2].

\section{Effect of Ototoxicity Monitoring on Veterans: Case Studies}

Below, we describe ototoxicity in two patients seen as part of our research on ototoxicity monitoring at the Portland VAMC. For these individuals, chemotherapy treatments resulted in hearing shifts before the treatment was completed. One patient opted to change medication; the other patient's disease necessitated that he continue with the planned regimen. Associated audiometric data for the patient described in case study 1 are presented in the "Clinical Objectives for COMP-VA" section.

\section{Case Study 1}

This patient is a 63 yr-old male treated for squamous cell carcinoma of the right base of tongue (stage IVa) with lymph node involvement. The planned chemotherapy regimen included three doses of cisplatin at $100 \mathrm{mg} / \mathrm{m}^{2}$ on days 1, 22, and 43 with concurrent head and neck radiation over $7 \mathrm{wk}$. An audiometric baseline evaluation was completed, at which time he presented with normal hearing through $4,000 \mathrm{~Hz}$ sloping to a moderate loss of hearing at 6,000 and $8,000 \mathrm{~Hz}$ in both ears. His high frequency limit of hearing was 12,500 and $11,200 \mathrm{~Hz}$ in the right and left ears, respectively. He reported bilateral intermittent tinnitus that was not bothersome. Otoscopy 
and tympanometry were normal bilaterally. Three weeks after his first dose (cumulative dose $=190 \mathrm{mg}$ ) and just before his second dose, a monitor evaluation revealed an ASHA-significant hearing change (average $+10 \mathrm{~dB}$ shift) at 10,000 to $12,500 \mathrm{~Hz}$ in the right ear with normal otoscopy and tympanometry. The left ear SRO remained unchanged, as was his baseline tinnitus. A "screen failure" follow-up audiogram indicated the hearing shift was confined to the ultra-high frequencies in one ear. Oncology was notified of these early unilateral hearing changes outside the speech frequency range and their decision was to continue with the second cisplatin dose. Three weeks after his second dose and just before infusion with his third dose (cumulative dose $=380 \mathrm{mg}$ ), ASHA-significant hearing change in the right ear expanded to include the 4,000 to $12,500 \mathrm{~Hz}$ frequency range (average $+25 \mathrm{~dB}$ shift) with no response to tones above $10,000 \mathrm{~Hz}$. There was a concomitant $+25 \mathrm{~dB}$ average shift in hearing in the left ear from 4,000 to $11,200 \mathrm{~Hz}$, and middle ear function remained normal bilaterally. He now reported more frequent tinnitus. Again, the oncology team was notified of the test results. At this point, the oncology nurse practitioner and audiologist together counseled the patient regarding the potential for further hearing changes if he continued treatment with cisplatin versus the option of changing to a less toxic chemotherapy drug, but with an undetermined effect on treatment efficacy. The audiologist explained options for aural rehabilitation, including the use of hearing aids. The oncology team was encouraged by the efficacy of treatment to this point. This patient-centered care approach involving both oncology and audiology resulted in the patient having the knowledge to make an informed decision regarding his care. He chose to preserve his hearing, and the oncologist changed his chemotherapy drug to carboplatin, a chemotherapy drug associated with less toxicity, for his last dose. The patient underwent imaging 3 mo following his last treatment, which indicated the cancer that had spread to the lymph nodes was no longer present, and endoscopy revealed the base of tongue primary cancer had regressed completely. Audiological follow-up with the patient was remarkable for otalgia, a retracted tympanic membrane, and worsening hearing loss in the right ear. His left ear remained stable. He was referred to otolaryngology, where a pressure equalization tube was placed in the right ear due to eustachian tube dysfunction secondary to radiation therapy.

\section{Case Study 2}

This patient is a $58 \mathrm{yr}$-old male treated for squamous cell carcinoma of the right tonsil (stage IVa). The planned chemotherapy regimen included three doses of $100 \mathrm{mg} / \mathrm{m}^{2}$ of cisplatin on days 1, 22, and 43 with concurrent head and neck radiation over $7 \mathrm{wk}$. At the audiometric baseline evaluation, he presented with normal hearing through $2,500 \mathrm{~Hz}$ sloping to a moderately-severe hearing loss at $8,000 \mathrm{~Hz}$ with a high-frequency limit of hearing of $10,000 \mathrm{~Hz}$ in both ears. He reported bilateral low-level constant tinnitus. Otoscopy and tympanometry were within normal limits. Following his first dose of cisplatin (cumulative dose $=200 \mathrm{mg}$ ), he became neutropenic, which resulted in a $2 \mathrm{wk}$ delay in his cisplatin treatment. Before the second dose of cisplatin, the audiologist detected ASHA-significant hearing changes from 3,000 to $10,000 \mathrm{~Hz}$ in the right ear and 3,000 to $8,000 \mathrm{~Hz}$ in the left ear in the presence of normal otoscopy and tympanometry. The largest hearing shift was at $3,000 \mathrm{~Hz}$ $(+30 \mathrm{~dB}$ average shift in the left and right ears) with lesser changes $(+10$ to $+15 \mathrm{~dB}$ shifts $)$ seen in the higher frequencies $(\geq 4,000 \mathrm{~Hz})$. The patient reported using a power drill on concrete without hearing protection following his first dose of cisplatin, despite being counseled by the audiologist to avoid noise overexposure. Because of concern that the noise exposure exacerbated the cisplatin effects, the patient was counseled again and provided new hearing protection. The oncology team was notified of the hearing changes but counseled the patient to proceed with cisplatin treatment to give him the best radiation therapy support. He was prepared for the possibility that his hearing could worsen. Following the second dose of cisplatin (cumulative dose $=400 \mathrm{mg}$ ), therapy was complicated by an $8 \mathrm{~d}$ hospital admission for neutropenia, fever, and chemotherapy-induced nausea/vomiting. The 2 wk delay in day 22 of treatment complicated by the recent hospital admission resulted in the discontinuation of day 43 treatment. A postexposure follow-up examination was conducted 1 mo following the second dose of cisplatin. At that time, ASHA-significant hearing changes were noted from 2,000 to $10,000 \mathrm{~Hz}$ in the right ear and 2,000 to $8,000 \mathrm{~Hz}$ in the left ear compared with baseline measurements in the presence of normal otoscopy and tympanometry. He denied any new noise exposure. Results indicated hearing loss had progressed following the second cycle to include hearing changes at $2,000 \mathrm{~Hz}$ in both ears averaging $+48 \mathrm{~dB}$ shifts. Hearing shifts $\geq 3,000 \mathrm{~Hz}$ remained stable in both ears. The patient now 
reported increased difficulty understanding speech, especially women and children, but denied other otologic complaints including aural fullness and changes in baseline tinnitus. A repeat audiological examination was conducted again 2 mo following cessation of cisplatin treatment (approximately $6 \mathrm{wk}$ following cessation of radiation therapy) and confirmed continued hearing changes through 2,000 Hz. Following treatment, he presents with a fairly flat moderately severe to severe hearing loss beginning at $2,000 \mathrm{~Hz}$. He feels that his hearing has significantly deteriorated since treatment and has been fit with bilateral hearing aids. His most recent examination by oncology shows that he is disease free.

\section{Clinical Objectives for COMP-VA}

Many oncologists strive to provide patient-centered chemotherapy treatment. The case studies just presented illustrate how ototoxicity monitoring information can influence counseling, treatment decisions, and utilization of posttreatment audiological services for individual patients. Below, we outline six clinical objectives that can be met using the COMP-VA program. We envision that these clinical objectives would be tailored to the needs and preferences of the audiologists, oncology teams, and individual patients.

1. Pretreatment ototoxicity risk assessment to demonstrate the anticipated hearing loss in the conventional frequency range as a result of the prescribed cisplatin dosing schedule.

2. Screening for early hearing changes to identify any ASHA-significant hearing shift within each patient's individualized SRO, as measured directly using puretone threshold testing or estimated using DPOAE testing.

3. Screening for outer hair cell dysfunction DPOAEs to identify early, potentially preclinical damage.

4. Screen failure follow-up testing to determine the extent that hearing changes include frequencies in the conventional audiometric frequency range as measured directly using pure-tone threshold testing or estimated using DPOAE testing.

5. Screening for tinnitus to determine whether the drug treatment is instigating or exacerbating tinnitus and the need for a tinnitus management referral.

6. Patient and provider education about ototoxicinduced hearing and tinnitus, synergistic effects of ototoxins and noise overexposure, and rehabilitative solutions to hearing loss and tinnitus.
The Table summarizes the COMP-VA clinical objectives pertinent to identifying and monitoring changes in hearing and outer hair cell function (objectives 1-4, listed above). The objectives are described in terms of the stakeholders requiring the data (who), the information being gathered (what), point in treatment to employ the test (when), basis for interpreting the results (how), and the evidence base supporting the test. Tinnitus screening and educational clinical objectives (objectives 5 and 6 , respectively) are not included in the table.

\section{Stakeholders}

Cancer treatment and management is obviously complex. Establishing the clinical objectives for ototoxicity monitoring collaboratively with the oncology team helps ensure that hearing results will be used in combination with other routine toxicity monitoring to affect patient care. Additionally, both the oncology team and the patient are more likely to support assessments of ototoxic symptoms and act on the results and recommendations if they are involved in developing individualized ototoxicity monitoring goals. An overarching goal of the COMPVA program is to form team relationships among audiology and oncology, thereby improving communication and coordination of care between these services for the benefit of Veterans and their families. We begin by defining the roles of the major program stakeholders.

\section{Audiologist}

In general, it is the responsibility of the audiologist to provide quality audiological care and to support the hearing healthcare needs of patients. Within the context of an ototoxicity monitoring program, this includes educating the patient about the potential for cisplatin to cause or exacerbate hearing loss and tinnitus. While the oncology team mentions the potential for hearing shift during treatment along with a litany of other toxicities, the audiologist is best able to provide information about the level of risk of hearing shift, factors that increase or decrease the risk, and options for rehabilitation should hearing shifts manifest. Additionally, it is the responsibility of the audiologist to emphasize the importance of using hearing protection and avoiding loud noise during and following treatment. This was illustrated in one of the case examples provided. Further, the audiologist is ideally positioned to understand how the pre-treatment hearing status or treatment-related change in the hearing status of any particular patient might affect that patient's ability to 
Table.

Description of proposed comprehensive ototoxicity monitoring program for Department of Veterans Affairs (COMP-VA) clinical objectives $1-4$. Table includes stakeholders in program (who), description of objective (what), implementation time (when), interpretation (how), and relevant citations (evidence base).

\begin{tabular}{|c|c|c|c|c|c|}
\hline Objective & Who: Stakeholder & $\begin{array}{l}\text { What: Description of } \\
\text { Clinical Objective }\end{array}$ & $\begin{array}{l}\text { When: Timing } \\
\text { \& Patient Status }\end{array}$ & $\begin{array}{l}\text { How: Basis for } \\
\text { Interpretation }\end{array}$ & $\begin{array}{c}\text { Evidence Base: } \\
\text { Relevant Citation(s) }\end{array}$ \\
\hline 1 & $\begin{array}{l}\text { Patient, audiologist, } \\
\text { oncologist }\end{array}$ & $\begin{array}{l}\text { Pretreatment risk } \\
\text { assessment: Predict } \\
\text { speech frequency shift }\end{array}$ & $\begin{array}{l}\text { Pretreatment } \\
\text { counseling \& } \\
\text { planning }\end{array}$ & $\begin{array}{l}\text { Baseline behavioral } \\
\text { audiogram }(\leq 8 \mathrm{kHz}) \\
\& \text { prescribed cisplatin } \\
\text { dose }\end{array}$ & $\begin{array}{l}\text { Dille et al., } 2012 \text { [1]; } \\
\text { Dille et al., } 2012 \text { [39] }\end{array}$ \\
\hline 2 & Audiologist & $\begin{array}{l}\text { Screening for outer hair } \\
\text { cell dysfunction: Detect } \\
\text { early changes in } \\
\text { cochlear function }\end{array}$ & $\begin{array}{l}\text { Any monitoring } \\
\text { appointment }\end{array}$ & $\begin{array}{l}\text { Larger change in } \\
\text { DPOAE level than } \\
\text { reference limits }\end{array}$ & $\begin{array}{l}\text { McMillan et al., } \\
2013 \text { [40]; McMil- } \\
\text { lan et al. [under } \\
\text { review] }\end{array}$ \\
\hline 3 & $\begin{array}{l}\text { Audiologist, } \\
\text { oncologist }\end{array}$ & $\begin{array}{l}\text { Screening for early } \\
\text { hearing changes } \\
\text { (behavioral): Detect } \\
\text { shift in SRO }\end{array}$ & $\begin{array}{l}\text { Any monitoring } \\
\text { appointment for } \\
\text { responsive/reli- } \\
\text { able patient }\end{array}$ & $\begin{array}{l}\text { Audiologist or } \\
\text { patient-administered } \\
\text { identification of } \\
\text { ASHA-significant } \\
\text { thresholds changes }\end{array}$ & $\begin{array}{l}\text { Fausti et al., } 1999 \\
\text { [19]; Konrad-Martin } \\
\text { et al., } 2010 \text { [5] }\end{array}$ \\
\hline \multirow{3}{*}{4} & $\begin{array}{l}\text { Audiologist, } \\
\text { oncologist }\end{array}$ & $\begin{array}{l}\text { Screening for early } \\
\text { hearing changes (non- } \\
\text { behavioral DPOAEs): } \\
\text { Estimate shift in SRO }\end{array}$ & $\begin{array}{l}\text { Any monitoring } \\
\text { appointment for } \\
\text { patient unable to } \\
\text { take behavioral } \\
\text { hearing test }\end{array}$ & $\begin{array}{l}\text { Baseline behavioral } \\
\text { audiogram (SRO), } \\
\text { administered cisplatin } \\
\text { dose, \& DPOAE I/O } \\
\text { or DPOAE fine step }\end{array}$ & $\begin{array}{l}\text { Dille et al., } 2010 \text { [2]; } \\
\text { Reavis et al., } 2011 \\
\text { [23]; McMillan et al., } \\
2013 \text { [40] }\end{array}$ \\
\hline & $\begin{array}{l}\text { Patient, audiologist, } \\
\text { oncologist }\end{array}$ & $\begin{array}{l}\text { Screen failure follow- } \\
\text { up testing (behavioral): } \\
\text { Detect speech fre- } \\
\text { quency shift }\end{array}$ & $\begin{array}{l}\text { Upon screen fail } \\
\text { for responsive/ } \\
\text { reliable patient }\end{array}$ & $\begin{array}{l}\text { Behavioral audio- } \\
\text { gram }(\leq 8 \mathrm{kHz})\end{array}$ & \\
\hline & $\begin{array}{l}\text { Patient, audiologist, } \\
\text { oncologist }\end{array}$ & $\begin{array}{l}\text { Screen failure follow- } \\
\text { up testing (nonbehav- } \\
\text { ioral DPOAEs): Esti- } \\
\text { mate speech frequency } \\
\text { shift }\end{array}$ & $\begin{array}{l}\text { Upon screen fail } \\
\text { for patient unable } \\
\text { to take behavioral } \\
\text { hearing test }\end{array}$ & $\begin{array}{l}\text { DPOAE model of } \\
\text { behavioral audio- } \\
\operatorname{gram}(\leq 8 \mathrm{kHz})\end{array}$ & \\
\hline
\end{tabular}

communicate. The audiologist is responsible for ensuring that the patient can adequately hear important treatment information and to provide strategies for effective communication including assistive hearing devices, if needed. The audiologist will also act as an intermediary between the patient and medical team if the team expresses concern for whether the patient can hear conversation adequately. Finally, it is the responsibility of the audiologist to identify those patients who need posttreatment support to address their hearing loss and tinnitus, which may include the acquisition of hearing aids. Patients undergoing cisplatin chemotherapy encounter a complex array of medical, psychological, and social challenges. The audiologist could work with the patient to set up audiology appointments and check back with the patient if electronic medical records indicate the appoint- ment was not kept. All aspects of the audiologist's role just defined are consistent with ASHA accepted care guidelines [20] and AAA position statements [26] for ototoxicity monitoring. Providing real-time information about ototoxicity and aural rehabilitation to the patient and medical team helps to achieve audiological care that is consistent with these guidelines and promotes the highest possible posttreatment quality of life.

\section{Oncologist}

The oncologist has the complex goal of balancing treatment needs with side-effect management. Working together with the audiologist, the oncologist will gain greater insight into the adverse effects of cisplatin on communication and, through these interactions, will gain an understanding of the benefits and limitations of current 
rehabilitation options for the treatment of hearing loss and tinnitus. While hearing aids improve hearing dramatically, mostly by increasing the level of soft sounds in the environment, they do not fully recover hearing that is lost. Damage to outer hair cells from cisplatin changes above-threshold sound processing in a way that can reduce frequency contrasts important for distinguishing speech sounds and can narrow the range of tolerable sound levels. These problems can influence the ability of an individual to understand speech and obtain benefit from hearing aids. Further, a growing body of literature suggests that peripheral hearing loss leads over time to major changes in auditory brainstem structures and neurochemistry - changes that have been linked to impaired auditory temporal processing in animal models [35-37]. While it is difficult to determine using clinical methods the extent that frequency tuning, perceptual loudness growth, and temporal processing are impaired, it is clear that the speech signal processed by a hearing aid and then an impaired ear can be substantially degraded. Perhaps for this reason and/or because of barriers to health promotion that are not unique to hearing health, hearing aid use is only about 20 percent among the general population for those with hearing loss [38]. For professionals who are not audiologists, gaining a deeper understanding of the importance of preserving hearing, when possible, cannot be underestimated. Finally, the oncologist will be made aware of each patient's risk for hearing change based on pretreatment information and will be provided with real-time information about cisplatin ototoxicity at each treatment interval. Armed with this information, the oncologist can consider changing the drug to one that is less ototoxic, modify the drug dosage, discontinue treatment and, if no ototoxicity is noted, have greater confidence that hearing after treatment will be spared.

\section{Patient}

Family members often report that Veterans with hearing loss underestimate the amount of communication difficulty they have. The patient should be made aware that chemotherapy treatment may worsen their hearing during and sometimes months following treatment. For patients who already have a preexisting hearing loss, a further decrease in hearing from cisplatin may be very detrimental to communication. Combining severe illness with already compromised communication may lead to feelings of isolation and depression and potentially affect conversations pertaining to medical treatment planning.
When tumor response to cisplatin is good, but ototoxic side effects are increasing, we have seen patients and oncology teams opt to change the treatment regimen. For other patients, the primary goal may be to treat the cancer in the most aggressive way possible. Therefore, it is essential that the audiologist, oncologist, and patient work together as a team to ensure fully informed decisions relating chemotherapy treatment and its ototoxic side effects are made. We propose that rehabilitative options be discussed whenever ototoxic changes are found. Investments made by the audiologist in patient education may help the Veteran and family members to move more smoothly through cancer treatment. As they begin to consider future audiological services, they will be better prepared for various audiological outcomes.

\section{Clinical Objective 1: Pretreatment Ototoxicity Risk Assessment}

One clinical objective of an ototoxicity monitoring program is to obtain a measure of an individual patient's susceptibility to, or conversely tolerance for, ototoxic damage. This information is used for two fundamental purposes. First, it can be used by oncology and audiology to predict, on an individual patient-ear basis, the dose at which a Veteran is most likely to have a significant hearing shift. This represents an extremely valuable tool for patient counseling. Armed with this individualized information, the audiology/oncology team can tailor discussions about the potential for cisplatin ototoxicity. Second, this information will provide oncology with a truly individualized treatment-ototoxicity profile, which could potentially assist with treatment planning that considers the balance between a curative approach and quality of life outcomes following treatment. Finally, this type of evidence-based approach provides some guidance for the allocation of audiological resources. For example, this model indicates that some patients with particularly poor hearing might require less frequent monitoring than every dose because they are unlikely to have hearing shift until cisplatin cumulative dose rises to high levels.

We propose the oncologist be provided a series of prediction audiograms using the planned cisplatin dosing regimen (dashed lines). The patient's actual baseline audiogram (solid line) would be provided on the same graph with gray shading indicating the "speech banana." Figure 2 shows data using this format from case study 1 . This model of conventional frequency pure-tone thresholds uses cisplatin dose and threshold information from the 


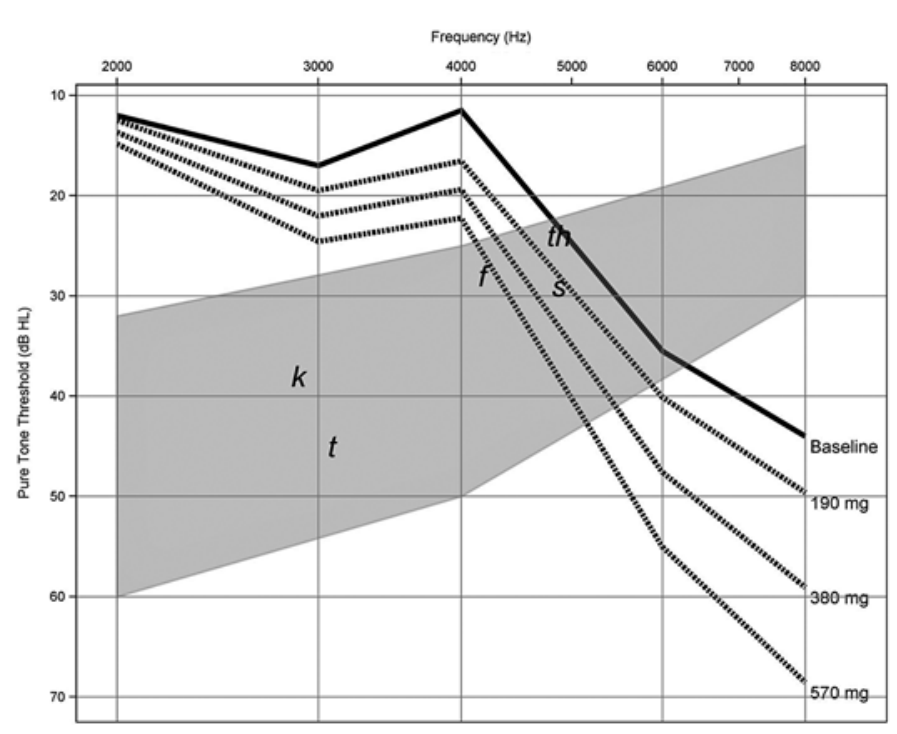

Figure 2.

Pretreatment risk assessment audiograms using threshold information from case study 1 . Series of prediction audiograms were generated using planned cisplatin dosing regimen (dashed lines) and patient's actual baseline audiogram (solid line) in decibels hearing level ( $\mathrm{dB} \mathrm{HL}$ ) shown as function of test frequency. Gray shading indicates "speech banana" with phonemes. This model of conventional frequency thresholds yielded overall accuracy of 4.9 to $8.0 \mathrm{~dB}$ prediction error.

preexposure audiogram provided by the audiologist. The model yielded an overall accuracy of 4.9 to $8.0 \mathrm{~dB}$ prediction error when tested against actual pure-tone threshold shifts observed in the functional speech frequency range [39]. Since most practitioners and patients remain most interested in changes that might occur in the future should treatments continue, future work will extend the predictive model throughout the conventional audiogram $(\leq 2,000 \mathrm{~Hz})$.

\section{Clinical Objective 2: Behavioral Screening for Early Hearing Changes}

For the purposes of COMP-VA, the screening for early hearing changes identifies any ASHA-significant pure-tone threshold shifts within each patient's individualized SRO measured using 1/6th-octave frequency increments. This behavioral protocol of the seven highest frequencies heard by an individual patient in each ear is operationally defined by a pure-tone threshold no higher than $100 \mathrm{~dB}$ SPL. The SRO is not a set frequency range that is tested on everyone. Rather the range varies across patients depending on their preexposure hearing ability, but in most Veterans includes some extended high frequencies $(>8,000 \mathrm{~Hz})$. The rationale is that this approach will sometimes allow chemotherapy treatment to be reconsidered before a handicapping hearing loss occurs, which is generally considered one that affects frequencies at and below about $4,000 \mathrm{~Hz}$. Past reports suggest that this individualized SRO approach is fast, yet captures early ototoxic changes. Each patient's individualized (by ear) SRO can be monitored for signs of hearing loss progression over many doses by expanding the range when thresholds can no longer be obtained at a limit of $100 \mathrm{~dB}$ SPL.

A graph of SRO thresholds for each ear that includes each monitoring visit (designated by the corresponding cumulative cisplatin dose) will be provided. Figure 3 shows data using this format for a single ear from case study 1 . SRO thresholds are provided in decibels SPL as a function of frequency. The bold line indicates the baseline evaluation while the dotted line indicates the evaluation associated with $190 \mathrm{mg}$ of cisplatin. The gray line indicates a monitoring result with ASHA-significant threshold shifts, prompting an examination of the conventional audiometric frequency range. Otoscopy and tympanometry results are used to help rule out a conductive component to the loss.

\section{Clinical Objective 3(a): Screening for Outer Hair Cell Dysfunction}

A graph of DPOAE level shifts superimposed on our newly developed test-retest reference limits will be provided to the audiologist [40]. The graph will indicate any changes in the number of valid responses at the monitor test compared with baseline, as a function of $\mathrm{f}_{2}$ frequency and dose. Figure 4 shows data using this format from the patient presented in case study 1, with DPOAE level in $\mathrm{dB}$ change from baseline as a function of $\mathrm{f}_{2}$ frequency. This patient had a DPOAE level decrement that was greater than the test-retest reference limits (gray fill) following his initial cumulative cisplatin dose of $190 \mathrm{mg}$ (black line) at the highest frequencies. There was also a large increment (DPOAE amplitude increased) at this early dose at around 3,000 $\mathrm{Hz}$. This change preceded any significant hearing changes, consistent with the view that DPOAEs provide early evidence of changes in cochlear health. Experience has shown that increments as well as decrements can be indicative of damage and a better understanding of the basis for the increments can be 


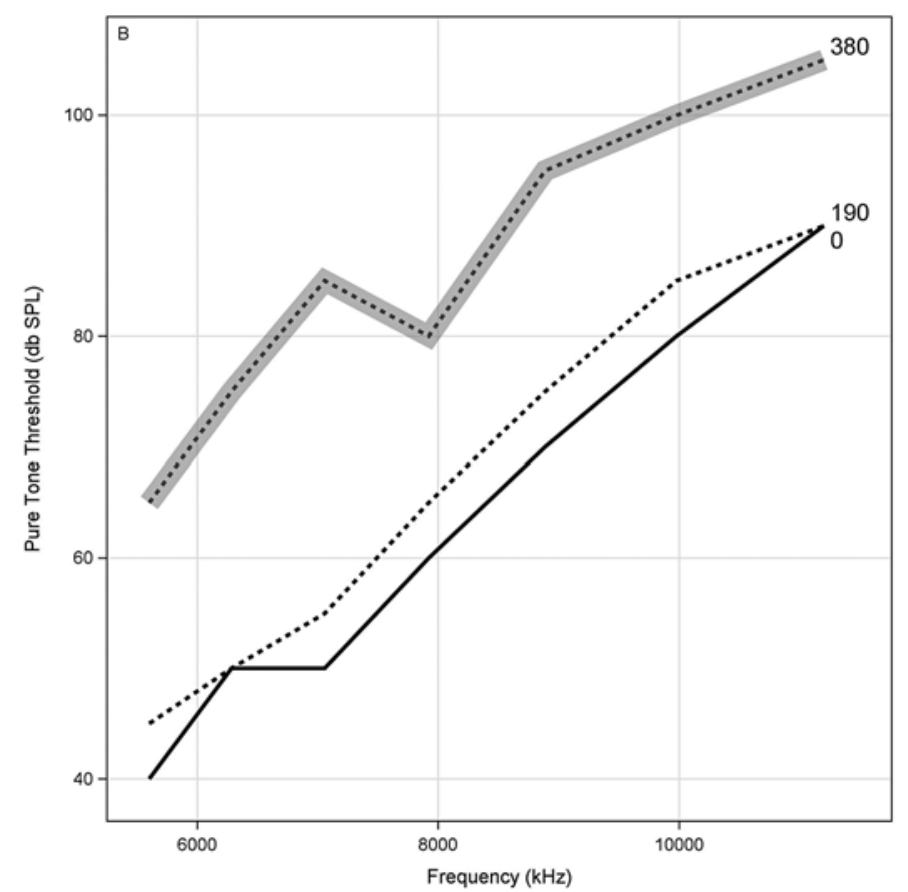

Figure 3.

Behavioral screening for early hearing changes using sensitive range for ototoxicity (SRO) protocol obtained from case study 1. SRO thresholds are provided in decibels sound pressure level (dB SPL) as function of frequency. Bold line indicates baseline evaluation, while dotted line indicates evaluation associated with $190 \mathrm{mg}$ of cisplatin. Gray dotted line indicates monitoring result with American Speech-Language-Hearing Association significant threshold shifts, prompting examination of conventional audiometric frequency range. Otoscopy and tympanometry results are used to help rule out conductive component to loss.

obtained by DPOAE source separation [41], which is possible to do if DPOAEs are collected using a fine $\mathrm{f}_{2^{-}}$ step DPOAE-gram protocol. At a cumulative dose of 380 $\mathrm{mg}$ (dotted line), DPOAEs had decreased over a wide range of frequencies, with decrements at several frequencies extending beyond the reference limits. To be interpreted as valid responses capable of providing monitoring data, DPOAEs levels must be greater than the combined noise and distortion by at least $6 \mathrm{~dB}$ and tympanometry results must be normal. Downward pointing triangles at the bottom of the graph indicate frequencies that were valid at baseline but failed the signal-to-noise ratio criteria because of a low amplitude response at a given monitoring visit (indicated by row). Upward pointing triangles show frequencies that gained a response or

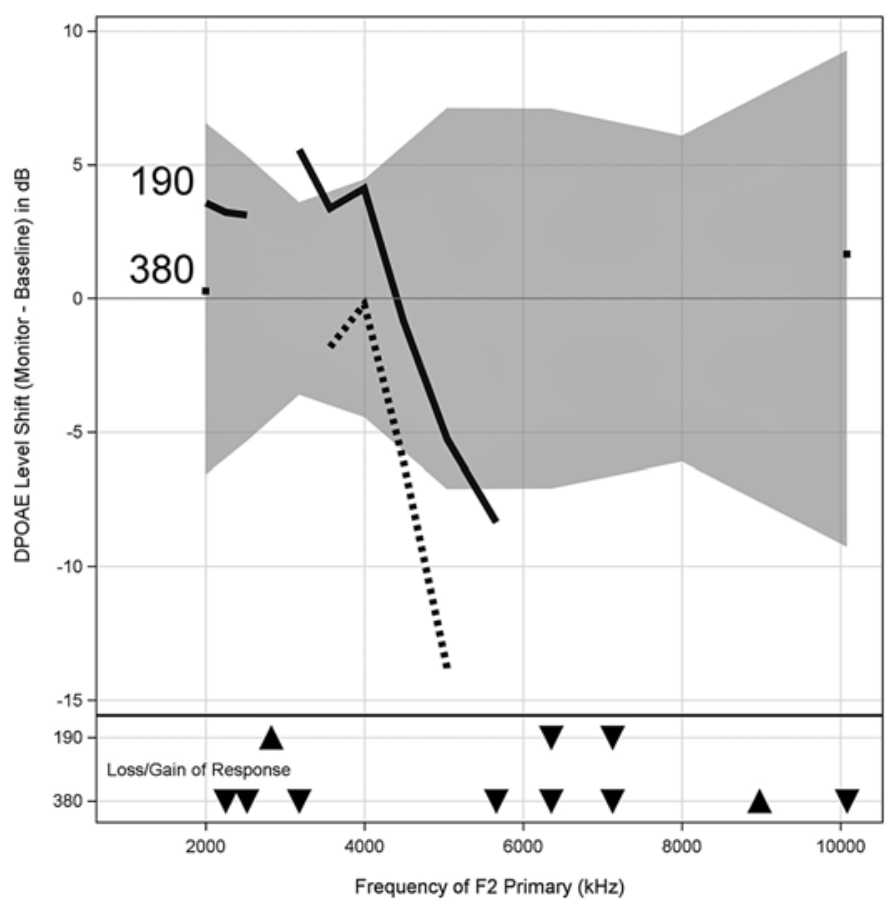

Figure 4.

Data from case study 1 in which distortion-product otoacoustic emission (DPOAE) level change (in decibels) from baseline is shown as function of $f_{2}$ frequency measured in fine (1/48octave) frequency steps. Test-retest reference limits obtained using same test protocol but on similarly aged subjects with no exposure to cisplatin are shown (gray fill). DPOAE level decrement was greater than test-retest at highest frequencies following initial cumulative cisplatin dose of $190 \mathrm{mg}$ (black line). Note also large increment (amplitude increased) at around $3,000 \mathrm{~Hz}$. At cumulative dose of $380 \mathrm{mg}$ (dotted line), DPOAEs decreased over wide range of frequencies, with decrements at several frequencies extending beyond reference limits. Downward pointing triangles at bottom of graph indicate frequencies valid at baseline but were low amplitude response at monitoring visit (indicated by row). Upward pointing triangles show frequencies that gained response or showed increment beyond reference limits compared with baseline test. Otoscopy and tympanometry results are used to help rule out conductive component to loss.

showed an increment beyond the reference limits compared with the baseline test.

\section{Clinical Objective 3(b): Nonbehavioral (DPOAE) Screening for Early Hearing Changes}

ASHA-significant pure-tone threshold shifts within the SRO can also be estimated using our validated multivariate algorithm combining DPOAE test-retest data with 
information from the baseline hearing test and the cumulative cisplatin dose, the ototoxicity risk assessment. COMP-VA will incorporate our best-performing DPOAE model [42], which uses data collected from DPOAE I/O functions near the DPOAE high-frequency limit of a valid response. Figure 5 shows data obtained in this manner with DPOAE output level plotted as a function of stimulus input level (L2). Data are presented for the same ear as shown previously from case study 1 . Each curve in the figure corresponds to a monitor visit with the cumulative dose indicated. Any measurement that fails to meet criteria for a valid response (level must be greater than the combined noise and distortion by at least $6 \mathrm{~dB}$ ) is indicated by an " $\mathrm{X}$ " on the figure corresponding to that input level. The gray line identifies the monitor visit at which an estimated ASHA-significant threshold shift occurs within the SRO, which would prompt an examination of the conventional audiometric frequency range. Since these data are from a research subject, we have both behavioral and DPOAE data at each monitoring visit. In this case, the DPOAE algorithm correctly identified the ASHA-significant hearing shift at $380 \mathrm{mg}$. Otoscopic and tympanometric results were used to rule out a conductive component to the loss.

Also evident in Figure 5 are substantial changes in DPOAE threshold at input levels from 35 to $45 \mathrm{~dB}$ SPL with the patient's first dose $(190 \mathrm{mg})$. These DPOAE input level changes accompanied hearing changes that were not clinically significant $(\leq 5 \mathrm{~dB})$, which simultaneously shows the potential value of DPOAEs as an early warning of imminent hearing change and illustrates the clinical utility of our multivariate model. In the absence of the model, it would be very difficult to know whether the observed DPOAE changes at $190 \mathrm{mg}$ were significant with respect to functional hearing.

At this point, DPOAE I/O level changes identify a change in the SRO frequencies capitalizing on early detection. A natural extension of this approach we intend to develop is a DPOAE model of behavioral thresholds in the speech frequency range.

\section{Clinical Objective 4: Screen Failure Follow-Up Testing}

Screen failures (for screening using behavioral SRO testing, DPOAE-gram testing or DPOAE I/O function testing) require the audiologist to complete a test of air conduction thresholds within the conventional frequency range when the patient is able to provide a reliable test. (Bone conduction thresholds are also tested at this point if tympanometry is abnormal.) This additional testing is

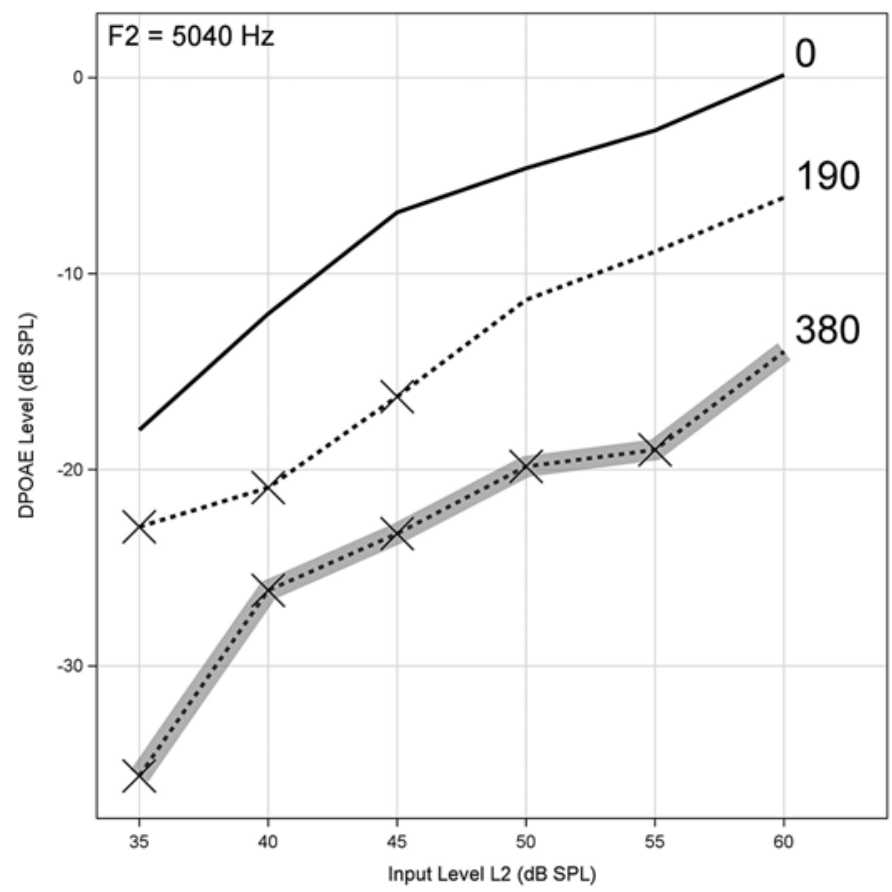

Figure 5.

Distortion-product otoacoustic emission (DPOAE) level plotted as function of L2 stimulus input level for $\mathrm{f}_{2}$ of $5,040 \mathrm{~Hz}$ from case study 1 stratified by cumulative drug dose. Measurements failing to meet criteria for valid response of $+6 \mathrm{~dB}$ greater than combined noise and system distortion are indicated by " $X$ " on figure corresponding to that input level. Gray line identifies monitor visit at which estimated American Speech-LanguageHearing Association-significant threshold shift will occur within sensitive range for ototoxicity using ototoxicity risk assessment. DPOAE level changes at cisplatin dose of $190 \mathrm{mg}$ to inputs of 35-45 dB sound pressure level (SPL) were not clinically significant $(\leq 5 \mathrm{~dB})$. Otoscopic and tympanometric results were used to rule out conductive component to loss.

done to determine the extent that hearing changes include speech frequencies and to rule out or quantify any conductive contribution to the loss. The main reason for this is that oncologists typically are not willing to withhold or change treatment based on hearing loss that does not threaten speech communication. It is not clear at this point whether oncologists treating cancer in adults would be willing to change treatment based on a physiological measure of ototoxicity such as a DPOAE.

Screen failure follow-up testing is best done at the same time as the positive test results are obtained. A COMP-VA graph will show conventional frequency thresholds for each ear corresponding to each monitoring 
visit in which this test was done, generally at baseline and following a screen failure. Figure 6 shows data using this format with the monitor visit designated by the corresponding cumulative cisplatin dose. Data are for the same ear previously shown for case study 1 . A comparison of Figure 6 with Figure 2 reveals close correspondence between the subject's predicted and actual loss at $380 \mathrm{mg}$. The chemotherapy regimen was changed from cisplatin to carboplatin following this hearing change based on input from the oncology team, audiologist, and patient. Data are collected in SPL and converted to show conventional frequency thresholds in decibels hearing level (HL) in order to be consistent with a typical clinical audiogram. Research data are shown for conventionalfrequency thresholds tested only down to $2,000 \mathrm{~Hz}$; however, we propose testing down to $500 \mathrm{~Hz}$ if possible, depending on ambient noise conditions on the ward.

\section{Other Elements of COMP-VA}

\section{Tinnitus Ototoxicity Monitoring Interview}

The Tinnitus Ototoxicity Monitoring Interview (TOMI) (Appendix, available online only) is designed to be given at the baseline visit and at each monitor visit in which the patient reports a change in tinnitus. The TOMI was developed by Dr. James Henry as a clinical tool to detect tinnitus onset or changes in the tinnitus percept during treatment. Portions of the TOMI were adapted from the TRT Initial Interview [43]. The TOMI is a 1-page instrument that can be completed within minutes. Ideally, the TOMI should be administered by an audiologist or otolaryngologist. However, because it is fully scripted, the TOMI can also be administered by a nurse or other healthcare professional who may not be familiar with clinical tinnitus issues, in which case the patient's responses should be reviewed by an audiologist or ear, nose, and throat physician.

\section{Testing on Oncology Ward Using COMP-VA}

Hospital noise comes from multiple sources that all combine into an overall ambient noise level on the treatment unit. The equivalent A-weighted noise level $\left(\mathrm{LA}_{\mathrm{EQ}}\right)$ has been measured in hospital wards and reported to be in the range of 40 to $46 \mathrm{~dB}$ with instantaneous peaks $\left(\mathrm{LC}_{\mathrm{PK}}\right)$ exceeding $90 \mathrm{~dB}$ [44]. Gordon et al. reported that Portland VAMC oncology ward noise measurements in 1/3-octave frequency bands did not exceed $37.5 \mathrm{dBA}$ at $500 \mathrm{~Hz}, 35 \mathrm{dBA}$ at $1,000 \mathrm{~Hz}, 36 \mathrm{dBA}$ at

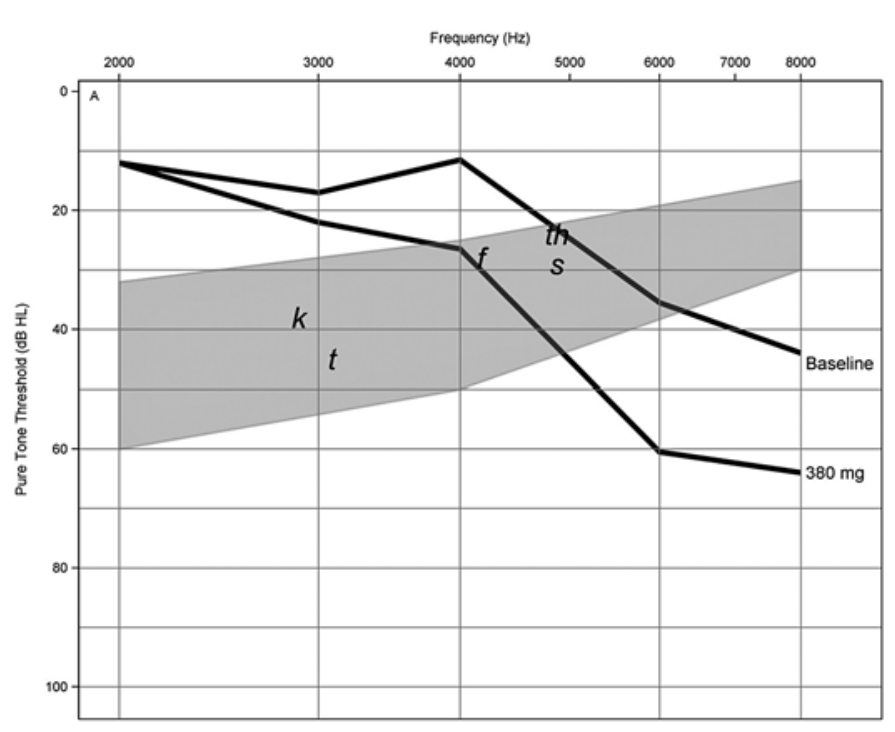

Figure 6.

Screen failure follow-up testing done at same time as hearing change to determine extent that hearing changes include frequencies within conventional audiometric frequency range. This graph, similar to an audiogram, plots conventional frequency $(\leq 8,000 \mathrm{~Hz})$ as function of audiometric threshold using data from case study 1 , stratified by cumulative drug dose. Comparison of Figure 6 with Figure 2, using same ear, reveals close correspondence between subject's predicted and actual loss at $380 \mathrm{mg} . \mathrm{HL}=$ hearing level.

$2,000 \mathrm{~Hz}$, and $32 \mathrm{dBA}$ at frequency bands greater than $3,000 \mathrm{~Hz}[45]$.

We applied MPANLs for audiometric test rooms [46] to the treatment unit for the purposes of COMP-VA. MPANLs are only reported up to $8,000 \mathrm{~Hz}$ and do not include MPANLs for the Sennheiser HDA-200 earphones, used with the OtoID. We have used the published Sennheiser passive attenuation data along with the published computational formula in the ANSI standard to develop estimates of MPANLs for the HDA-200 earphones from 125 to $16,000 \mathrm{~Hz}$ in $1 / 3$-octave frequency bands. In order to complete this calculation, we extrapolated data for frequencies above $8,000 \mathrm{~Hz}$. Figure 7 shows the results of our calculated MPANLs for the Sennheiser HDA-200 series earphones (circles), insert earphones (triangles), and hospital ward noise (asterisk) derived from Gordon et al. for frequencies $125-16,000 \mathrm{~Hz}$ [45]. The Sennheiser HDA-200 earphones are usable for threshold determination at frequencies above $1,000 \mathrm{~Hz}$, 


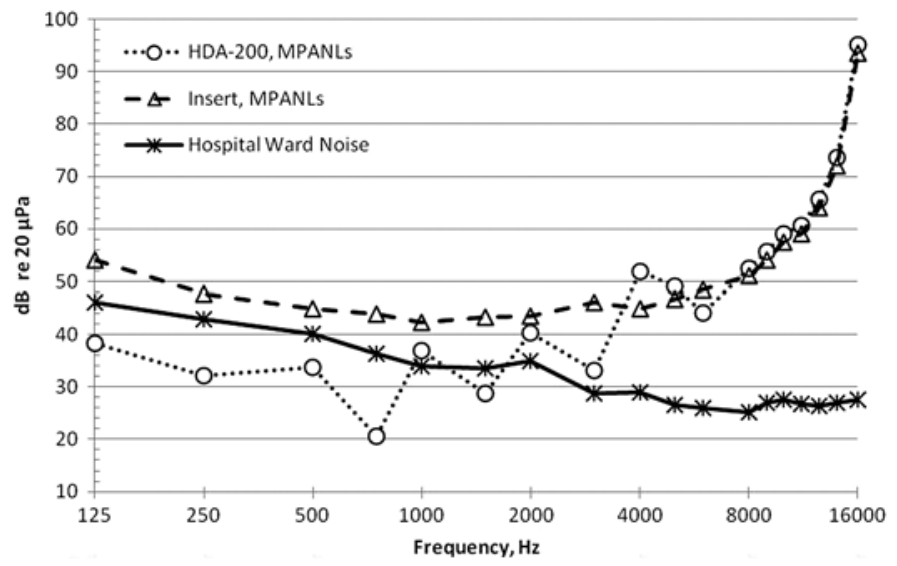

Figure 7.

Maximum permissible ambient noise levels (MPANLs) (in decibels re: $20 \mu \mathrm{Pa}$, American National Standards Institute S3.11999) for audiometric test room is shown as function of frequency when HDA 200 (circles) circumaural earphones and Etymotic ER2 (triangles) earphones are used. Hospital noise (asterisk) levels as function of frequency measured in 1/3octave frequency bands (Gordon et al., 2005 [45]) are also shown. Data show that reliable behavioral hearing thresholds can be obtained for frequencies above $2,000 \mathrm{~Hz}$ in most circumstances. In addition, room noise using OtolD is measured just before presentation of each tone as extra measure that noise levels in room are well controlled. Using insert earphones for collecting distortion-product otoacoustic emissions, all $\mathrm{f}_{2}$ frequencies can be used.

with the possible exception of 1,500 Hz. The insert earphones are useable at all test frequencies.

Furthermore, Gordon et al. reported on the use of circumaural and in-the-ear earphones for high frequency testing in the sound booth as well as on the hospital ward [45], reporting "test-retest reliability was found to be good for the KOSS circumaural earphones and the ER4-B insert earphones for both sound booth and hospital ward settings." Dille et al. reported on retest comparisons using the OtoID over $3 \mathrm{~d}$ on 40 subjects both young and older with and without hearing loss tested in a sound booth and on the hospital ward [17]. Ninety-five percent of all tests were within $\pm 5 \mathrm{~dB}$ with no systematic effect of location or subject. These results indicate that ward noise is not likely to interfere with collecting reliable threshold data when circumaural, supra-aural, or in-the-ear transducers are used and efforts are made to control or segregate ambient noise from the test environment. Further, the OtoID is capable of measuring room noise during testing and is designed to halt testing should noise in the room exceed preset limits.

Our DPOAE measurements are acquired using an insert probe with similar attenuation characteristics as insert earphones. MPANLs for DPOAE testing using an insert probe with sufficient averaging were estimated to be $\geq 55 \mathrm{dBA}$ for frequencies 2,000 to $6,000 \mathrm{~Hz}$ [47], which easily exceeds the ambient noise levels on the hospital ward [44-45]. The measurement of DPOAE data involves signal averaging in order to improve the signalto-noise ratios of the measurement. The number of averages that we take is adjusted in order to meet the desired noise floor of our measurement, which we have set to a level near the approximate level of our system distortion. This response-based averaging time is allowed to continue up to a predetermined halting time-out. An analysis of the average recording time for tests completed on the ward demonstrate that the desired noise floor is achieved in almost all cases before time-out is reached. This is an additional indicator that acceptable signal-to-ambient-noise ratios exist for our DPOAE testing in the hospital wards.

\section{OVERVIEW OF TESTING PROTOCOL AND SCHEDULE}

The proposed protocol for accomplishing objectives 1-4 is summarized schematically in Figure 8. The flowchart demonstrates how the COMP-VA monitoring protocol is used for detecting hearing and outer hair cell function changes. Pretreatment tests include otoscopy, tympanometry, and air conduction testing in conventional and extended high frequencies and SRO is determined for subsequent testing. Pretreatment risk calculations are done and used for educational and patient counseling purposes and, potentially, to help determine how to allocate hearing screening resources. Screening is ideally done just before each cisplatin infusion because changes in medication can be considered before the next dose. Screenings minimally include the SRO audiogram for responsive/reliable patients. A DPOAE-gram is also routinely done as a means to detect ototoxic changes early. Using the DPOAE-gram, DPOAE level changes are calculated relative to baseline measures and compared with our reference limits to determine whether the changes are greater than those attributable to normal test-retest variability. DPOAE I/O functions are reserved for patients who are too sick to provide a reliable hearing test. The 


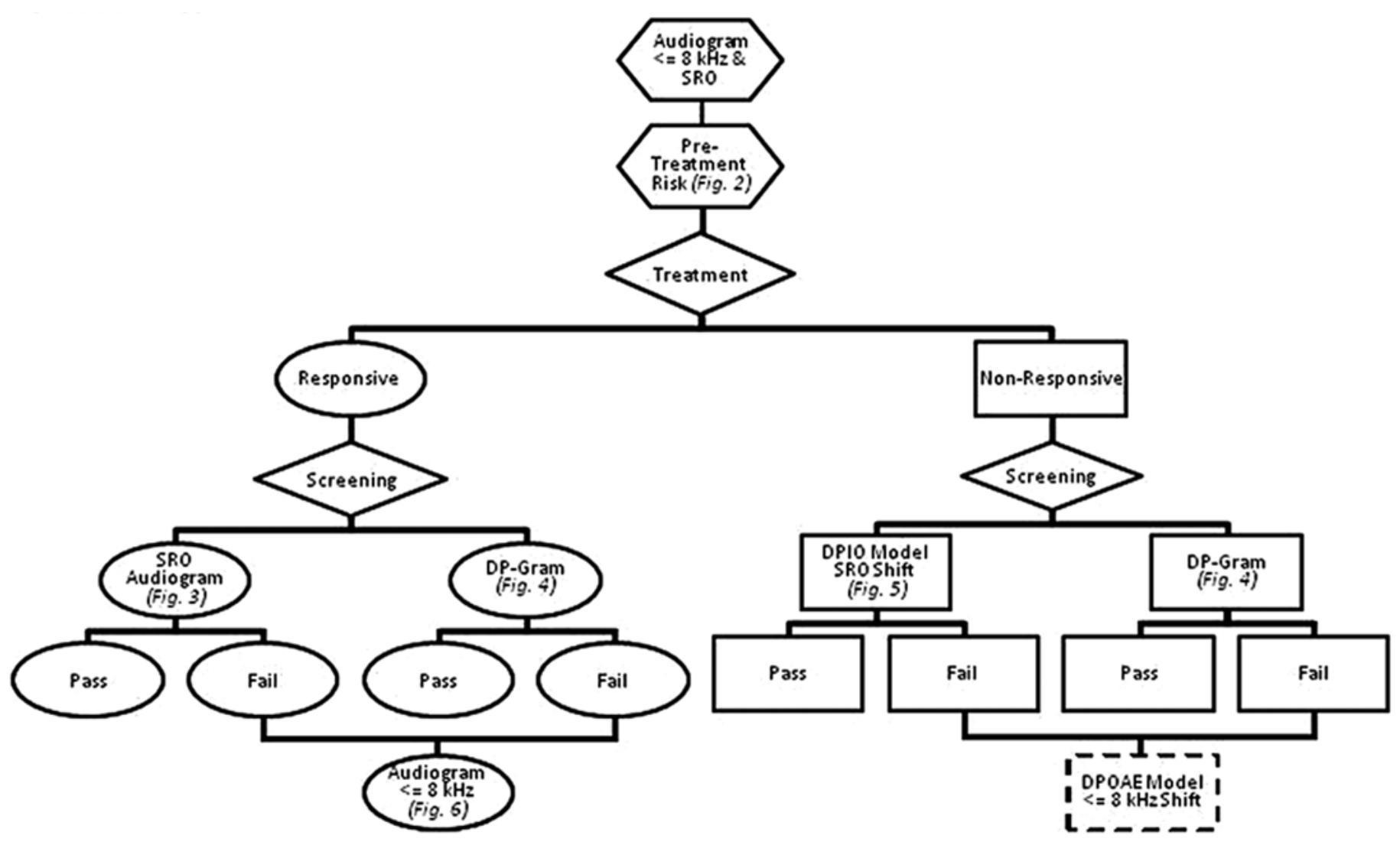

Figure 8.

Flowchart demonstrating how comprehensive ototoxicity monitoring program for Department of Veterans Affairs (COMP-VA) monitoring protocol is used for detecting hearing and outer hair cell function changes. Pretreatment tests include otoscopy, tympanometry, distortion-product otoacoustic emission (DPOAE) testing, and air-conduction testing in conventional and extended high frequencies, and sensitive range for ototoxicity (SRO) is determined for subsequent testing. From these tests, pretreatment risk calculation is done for educational and patient counseling purposes and may prove helpful when planning professional resources. Screenings minimally include SRO audiogram for responsive/reliable patients and DPOAE level plotted as a function of $\mathrm{f}_{2}$ frequency to detect early ototoxic changes. From DPOAE level plotted as a function of $\mathrm{f}_{2}$ frequency, DPOAE level changes are calculated relative to baseline measures and compared with our reference limits to determine whether changes are greater than those attributable to normal test-retest variability and DPOAE input-output functions (obtained from multiple levels) are reserved for patients who are too sick to provide reliable hearing test. Any screen failure requires follow-up testing, ideally done using behavioral hearing test.

DPOAE I/O function results are compared with the baseline test, and differences are used as inputs to our validated algorithm that identifies ASHA-significant shifts in the SRO. Any screen failure requires follow-up testing, ideally done using a behavioral hearing test.

COMP-VA advocates a testing regimen that includes a baseline (preexposure to cisplatin) evaluation, monitor evaluations before each cisplatin treatment, and a followup evaluation after the regimen is completed. It is often most convenient for the patient to conduct monitor evaluations during hydration just before the next cisplatin administration. This allows the oncologist at least some time to consider treatment changes should a hearing shift be discovered. Posttreatment regimen evaluations should be conducted in a timely fashion so that new or exacerbated audiological changes can be promptly addressed.

\section{DISCUSSION}

Both ASHA and AAA professional organizations provide position statements to the community of audiologists 
regarding the necessity and implementation of ototoxicity monitoring. Both position statements suggest that ototoxicity monitoring may not prove actionable to oncologists because cancer treatment objectives are in many cases immutable. This has not been our experience. Head and neck cancers tend to respond well to treatment while, in our Veteran population, lung cancer outcomes are not as favorable. Our oncologists consider the effect of communication deficits for Veteran survivors of cancer, as well as those in palliative care. They are interested in controlling treatment-related hearing loss and are willing to change treatment regimens or chemotherapeutic agents if it is medically responsible to do so when ototoxicity is discovered.

Since Veterans enter treatment with substantial hearing loss, ototoxicity monitoring has the potential to maintain quality of life following treatment. This is particularly true when the audiologist becomes an advocate for hearing preservation and rehabilitation. Months spent educating the Veteran patient on communication effects of hearing loss, avoidance of noise overexposure and, when necessary, timely access to assistive devices provide a rationale and motivation for hearing aids after treatment. ASHA suggests that audiologists are "ethically bound" to begin rehabilitation following treatment [20, p. $10]$. We enthusiastically agree and have built this into our program.

While COMP-VA is consistent with published ototoxicity monitoring recommendations in many ways, it differs from them in important ways. One particularly important distinction is that both organizational position statements suggest that the best monitoring approach is through a comprehensive auditory evaluation before monitoring (baseline). They advocate that testing should minimally include pure-tone air- and bone-conduction audiometry, otoscopy, tympanometry, and speech measures, with the rationale that to evaluate change, comprehensive evaluations are necessary. It is true that the functional effect of pure-tone change in the conventional frequencies will be expressed in speech results. However, it is unlikely that rehabilitation can be effectively addressed during treatment beyond an assistive device. Cancer treatment is fairly short-term, lasting sometimes only months. By the nature of its screening protocols, COMP-VA suggests postponing rehabilitation until treatment has been completed. However, we do agree that any testing from which comparisons will be made must be done at baseline. We advocate the time-efficient screen- ing SRO protocol at baseline combined with tympanometry and otoscopy to rule out ear disease and obstruction. If otoacoustic emission testing is anticipated during treatment, it also must be done at the baseline visit in order to have a basis for meaningful interpretation of the findings.

Guidelines from ASHA and AAA, published $15 \mathrm{yr}$ later, do not promote SRO testing; however, they do support the use of high frequency audiometry. The SRO omission may have been the result of the lack of instrument availability for $1 / 6$-octave testing at the time. Both sets of guidelines recognize that DPOAE testing is a sensitive measure of outer hair cell function that may be especially important for the testing of elderly and hearing impaired patients and that tinnitus information obtained during treatment ought to be methodically obtained, consistent with our recommendations.

Despite substantial evidence and professional guidelines for alleviating preventable hearing loss, ototoxicity monitoring remains underutilized in the VA. In 2008, a national survey of VA audiologists from approximately 40 medical centers confirmed several barriers to widespread implementation, with common responses being the lack of (1) appropriate instrumentation with which to perform ototoxicity testing in varied test locations; (2) access to specific, evidence-based protocols suitable for testing all patients regardless of illness; and (3) personnel resources to perform labor-intensive ototoxicity monitoring protocols.

COMP-VA attempts to address each of these barriers with clinically appropriate and efficient evidence-based protocols and instrumentation. The efficiencies we propose were designed to provide systematic monitoring throughout treatment, while conserving audiological resources, now a necessary feature when introducing a new clinical service to VA. Aspects of the program include the OtoID, a relatively low-cost, high-fidelity portable audiometer that has a wide test-frequency range, can test in fine frequency steps using manual or automated modes, and mitigates the potential influence of ambient noise on the test. Using evidence-based behavioral and objective test protocols, COMP-VA provides up-to-the-minute estimates of ototoxicity before the patient's next treatment, which allows for timely consideration of treatment changes. Proposed objective tests include methods for identifying outer hair cell changes and predicting audiogram changes using DPOAEs.

Further work to accomplish includes expanding the speech frequency prediction model to include frequencies 
below 2,000 Hz. Early identification of hearing shift is important. However, oncologists tell us that, in addition, they would like to know when communication will be significantly affected. This could provide them an opportunity to give additional doses that potentially bring the Veteran closer to a cure without significantly affecting quality of life following treatment.

A limitation to our current DPOAE-gram screening protocol is that the preliminary DPOAE reference limits do not extend testing into the time period that reflects cisplatin treatment, which often occurs over several months. This is a common problem among DPOAE test-retest variability studies, in which retests extend only out to approximately $15 \mathrm{~d}$. The ramification is that COMP-VA retest reference limits do not account for increases in variability over time. A model based on a meta-analysis of the published DPOAE test-retest variability is being developed that will extrapolate the variability estimates over longer durations.

Having DPOAE prediction indicators in a variety of important frequency intervals (extended high frequency and speech frequency) could lend confidence to an indication that hearing has changed and to a decision to continue to treat or to hold treatment pending behavioral hearing results. Planned research will therefore include DPOAE prediction of speech frequency change, as well as presence/absence of SRO shifts.

\section{CONCLUSIONS}

A successful ototoxicity monitoring program requires team relationships between audiology and oncology so that communication between services can be efficient. Monitoring that can be conducted almost exclusively chairside addresses issues of limited sound booth availability and the need to "add on" testing appointments, often required for ototoxicity monitoring programs. Presenting clear, reliable, easily understood and evidence-based test results will improve communication and trust within the team, thus enhancing therapeutic planning, coordination of care, and informed decision-making. Finally, having a robust ototoxicity monitoring program like COMP-VA in place also ensures that an audiologist who has developed a working relationship with the patient during multiple testing sessions is also able to provide posttreatment support and encouragement should rehabilitation become necessary after treatment.

\section{ACKNOWLEDGMENTS}

\section{Author Contributions:}

Obtained funding: D. Konrad-Martin, M. Dille.

Contributed to important intellectual content of study design:

D. Konrad-Martin, M. Dille, G. McMillan.

Collected data: M. Dille, K. M. Reavis, W. J. Helt.

Analyzed data: G. McMillan.

Wrote manuscript: D. Konrad-Martin, M. Dille, K. M. Reavis, W. J. Helt. Financial Disclosures: The authors have declared that no competing interests exist.

Funding/Support: This material was based on work supported by the VA RR\&D Service (grants C7113N, C7223R and C6373R) and the VA RR\&D NCRAR.

Additional Contributions: Portions of this work were presented at the 2011 Joint Defense Veterans Audiology Conference, San Diego, California, and the 2012 Association of VA Hematology/Oncology Conference, Omaha, Nebraska.

Institutional Review: This study reviews and reanalyzes data collected in previous studies, as indicated throughout the article. All subjects consented to participate (i.e., signed an informed consent form), and each study maintained approval from Portland VA Medical Center's Institutional Review Board.

Participant Follow-Up: The authors do not plan to inform participants of the publication of this study. However, participants have been encouraged to check the NCRAR Web site for updated publications.

\section{REFERENCES}

1. Dille MF, Wilmington D, McMillan GP, Helt WJ, Fausti SA, Konrad-Martin D. Development and validation of a cisplatin dose-ototoxicity model. J Am Acad Audiol. 2012;23(7):510-21. [PMID:22992258]

http://dx.doi.org/10.3766/jaaa.23.7.3

2. Dille MF, McMillan GP, Reavis KM, Jacobs P, Fausti SA, Konrad-Martin D. Ototoxicity risk assessment combining distortion product otoacoustic emissions with a cisplatin dose model. J Acoust Soc Am. 2010;128(3):1163-74.

[PMID:20815453]

http://dx.doi.org/10.1121/1.3473693

3. Reavis KM, Phillips DS, Fausti SA, Gordon JS, Helt WJ, Wilmington D, Bratt GW, Konrad-Martin D. Factors affecting sensitivity of distortion-product otoacoustic emissions to ototoxic hearing loss. Ear Hear. 2008;29(6):87593. [PMID:18753950]

http://dx.doi.org/10.1097/AUD.0b013e318181ad99

4. Fausti SA, Helt WJ, Phillips DS, Gordon JS, Bratt GW, Sugiura KM, Noffsinger D. Early detection of ototoxicity using 1/6th-octave steps. J Am Acad Audiol. 2003;14(8): 444-50. [PMID:14655957]

5. Konrad-Martin D, James KE, Gordon JS, Reavis KM, Phillips DS, Bratt GW, Fausti SA. Evaluation of audiometric threshold shift criteria for ototoxicity monitoring. J Am 
Acad Audiol. 2010;21(5):301-14, quiz 357. [PMID:20569665]

http://dx.doi.org/10.3766/jaaa.21.5.3

6. Fosså SD, de Wit R, Roberts JT, Wilkinson PM, de Mulder PH, Mead GM, Cook P, de Prijck L, Stenning S, Aaronson NK, Bottomley A, Collette L; European Organization for Research and Treatment of Cancer Genitourinary Group 30941; Medical Research Council Testicular Cancer Study Group TE20. Quality of life in good prognosis patients with metastatic germ cell cancer: A prospective study of the European Organization for Research and Treatment of Cancer Genitourinary Group/Medical Research Council Testicular Cancer Study Group (30941/TE20). J Clin Oncol. 2003;21(6):1107-18. [PMID:12637478] http://dx.doi.org/10.1200/JCO.2003.02.075

7. Dalton DS, Cruickshanks KJ, Klein BE, Klein R, Wiley TL, Nondahl DM. The impact of hearing loss on quality of life in older adults. Gerontologist. 2003;43(5):661-68. [PMID:14570962]

http://dx.doi.org/10.1093/geront/43.5.661

8. Schultz C, Goffi-Gomez MV, Liberman PH, Carvalho AL. Report on hearing loss in oncology. Braz J Otorhinolaryngol. 2009;75(5):634-41. [PMID:19893928] http://dx.doi.org/10.1590/S1808-86942009000500004

9. Schweitzer VG. Cisplatin-induced ototoxicity: The effect of pigmentation and inhibitory agents. Laryngoscope. 1993;103(4 Pt 2):1-52. [PMID:8464301] http://dx.doi.org/10.1288/00005537-199304000-00001

10. Arora R, Thakur JS, Azad RK, Mohindroo NK, Sharma DR, Seam RK. Cisplatin-based chemotherapy: Add highfrequency audiometry in the regimen. Indian $\mathrm{J}$ Cancer. 2009;46(4):311-17. [PMID:19749461] http://dx.doi.org/10.4103/0019-509X.55551

11. Laurell G, Viberg A, Teixeira M, Sterkers O, Ferrary E. Blood-perilymph barrier and ototoxicity: An in vivo study in the rat. Acta Otolaryngol. 2000;120(7):796-803. [PMID:11132710] http://dx.doi.org/10.1080/000164800750061624

12. Hellberg V, Wallin I, Eriksson S, Hernlund E, Jerremalm E, Berndtsson M, Eksborg S, Arnér ES, Shoshan M, Ehrsson H, Laurell G. Cisplatin and oxaliplatin toxicity: Importance of cochlear kinetics as a determinant for ototoxicity. J Natl Cancer Inst. 2009;101(1):37-47. [PMID:19116379] http://dx.doi.org/10.1093/jnci/djn418

13. Mukherjea D, Rybak LP. Pharmacogenomics of cisplatininduced ototoxicity. Pharmacogenomics. 2011;12(7):1039-50. [PMID:21787192]

http://dx.doi.org/10.2217/pgs.11.48

14. Riedemann L, Lanvers C, Deuster D, Peters U, Boos J, Jürgens H, am Zehnhoff-Dinnesen A. Megalin genetic polymorphisms and individual sensitivity to the ototoxic effect of cisplatin. Pharmacogenomics J. 2008;8(1):23-28.

\section{[PMID:17457342]}

http://dx.doi.org/10.1038/sj.tpj.6500455

15. Lee JE, Nakagawa T, Kim TS, Iguchi F, Endo T, Dong Y, Yuki K, Naito Y, Lee SH, Ito J. A novel model for rapid induction of apoptosis in spiral ganglions of mice. Laryngoscope. 2003;113(6):994-99. [PMID:12782811] http://dx.doi.org/10.1097/00005537-200306000-00015

16. Rybak LP. Mechanisms of cisplatin ototoxicity and progress in otoprotection. Curr Opin Otolaryngol Head Neck Surg. 2007;15(5):364-69. [PMID:17823555] http://dx.doi.org/10.1097/MOO.0b013e3282eee452

17. Dille MF, Jacobs PG, Gordon SY, Helt WJ, McMillan GP. OtoID: New extended frequency, portable audiometer for ototoxicity monitoring. J Rehabil Res Dev. 2013;50(7): 997-1006. [PMID:24301436]

http://dx.doi.org/10.1682/JRRD.2012.09.0176

18. Jacobs PG, Silaski G, Wilmington DJ, Gordon S, Helt WJ, McMillan GP, Fausti SA, Dille MF. Development and evaluation of a portable audiometer for high-frequency screening of hearing loss from ototoxicity in homes/clinics. IEEE Trans Biomed Eng. 2012;59(11):3097-3103.

[PMID:22801480] http://dx.doi.org/10.1109/TBME.2012.2204881

19. Fausti SA, Henry JA, Helt WJ, Phillips DS, Frey RH, Noffsinger D, Larson VD, Fowler CG. An individualized, sensitive frequency range for early detection of ototoxicity. Ear Hear. 1999;20(6):497-505. [PMID:10613387] http://dx.doi.org/10.1097/00003446-199912000-00005

20. American Speech-Language-Hearing Association. Guidelines for the audiologic management of individuals receiving cochleotoxic drug therapy [Internet]. Rockville (MD): ASHA; 2013. Available from: http://www.asha.org/policy/ GL1994-00003.htm

21. Vaughan NE, Fausti SA, Chelius S, Phillips D, Helt W, Henry JA. An efficient test protocol for identification of a limited, sensitive frequency test range for early detection of ototoxicity. J Rehabil Res Dev. 2002;39(5):567-74. [PMID:17642021]

22. Ress BD, Sridhar KS, Balkany TJ, Waxman GM, Stagner BB, Lonsbury-Martin BL. Effects of cis-platinum chemotherapy on otoacoustic emissions: The development of an objective screening protocol. Third place-Resident Clinical Science Award 1998. Otolaryngol Head Neck Surg. 1999;121(6):693-701. [PMID:10580222] http://dx.doi.org/10.1053/hn.1999.v121.a101567

23. Reavis KM, McMillan GP, Austin D, Gallun F, Fausti SA, Gordon JS, Helt WJ, Konrad-Martin D. Distortion-product otoacoustic emission test performance for ototoxicity monitoring. Ear Hear. 2011;32(1):61-74. [PMID:20625302]

24. Hallmark RJ, Snyder JM, Jusenius K, Tamimi HK. Factors influencing ototoxicity in ovarian cancer patients treated 
with Cis-platinum based chemotherapy. Eur J Gynaec Oncol. 1992;13(1):35-44.

25. Bokemeyer C, Berger CC, Hartmann JT, Kollmannsberger C, Schmoll H-J, Kuczyk MA, Kanz L. Analysis of risk factors for cisplatin-induced ototoxicity in patients with testicular cancer. Br J Cancer. 1998;77(8):1355-62.

[PMID:9579846]

http://dx.doi.org/10.1038/bjc.1998.226

26. American Academy of Audiology. American Academy of Audiology Position Statement and Clinical Practice Guidelines: Ototoxicity Monitoring [Internet]; Reston (VA); 2013. Available from: http://www.audiology.org/resources/ documentlibrary/Documents/OtoMonGuidelines.pdf

27. Fausti SA, Rappaport BZ, Frey RH, Henry JA, Phillips DS, Mitchell CR, Olson DJ. Reliability of evoked responses to high-frequency $(8-14 \mathrm{kHz})$ tone bursts. J Am Acad Audiol. 1991;2(2):105-14. [PMID:1768872]

28. Fausti SA, Frey RH, Henry JA, Olson DJ, Schaffer HI. Early detection of ototoxicity using high-frequency, toneburst-evoked auditory brainstem responses. J Am Acad Audiol. 1992;3(6):397-404. [PMID:1486202]

29. Arnold DJ, Lonsbury-Martin BL, Martin GK. High-frequency hearing influences lower-frequency distortion-product otoacoustic emissions. Arch Otolaryngol Head Neck Surg. 1999;125(2):215-22. [PMID:10037289]

http://dx.doi.org/10.1001/archotol.125.2.215

30. Sie KC, Norton SJ. Changes in otoacoustic emissions and auditory brain stem response after cis-platinum exposure in gerbils. Otolaryngol Head Neck Surg. 1997;116(6 Pt 1): 585-92. [PMID:9215367] http://dx.doi.org/10.1016/S0194-5998(97)70232-8

31. Hodges AV, Lonsbury-Martin BL. Hearing management. San Diego (CA): Singular Press; 1998.

32. Mauermann M, Uppenkamp S, van Hengel PW, Kollmeier B. Evidence for the distortion product frequency place as a source of distortion product otoacoustic emission (DPOAE) fine structure in humans. II. Fine structure for different shapes of cochlear hearing loss. J Acoust Soc Am. 1999;106(6):3484-91. [PMID:10615688] http://dx.doi.org/10.1121/1.428201

33. Konrad-Martin D, Neely ST, Keefe DH, Dorn PA, Cyr E, Gorga MP. Sources of DPOAEs revealed by suppression experiments, inverse fast Fourier transforms, and SFOAEs in impaired ears. J Acoust Soc Am. 2002;111(4):1800-1809. [PMID:12002864] http://dx.doi.org/10.1121/1.1455024

34. Konrad-Martin D, Reavis KM, McMillan GP, Dille MF. Multivariate DPOAE metrics for identifying changes in hearing: Perspectives from ototoxicity monitoring. Int $\mathrm{J}$ Audiol. 2012;51(Suppl 1):S51-62. [PMID:22264063] http://dx.doi.org/10.3109/14992027.2011.635713
35. Caspary DM, Milbrandt JC, Helfert RH. Central auditory aging: GABA changes in the inferior colliculus. Exp Gerontol. 1995;30(3-4):349-60. [PMID:7556513]

http://dx.doi.org/10.1016/0531-5565(94)00052-5

36. Willott JF, Milbrandt JC, Bross LS, Caspary DM. Glycine immunoreactivity and receptor binding in the cochlear nucleus of $\mathrm{C} 57 \mathrm{BL} / 6 \mathrm{~J}$ and $\mathrm{CBA} / \mathrm{CaJ}$ mice: Effects of cochlear impairment and aging. J Comp Neurol. 1997;385(3): 405-14. [PMID:9300767] http://dx.doi.org/10.1002/(SICI)10969861(19970901)385:3<405::AID-CNE5>3.0.CO;2-7

37. Wang H, Turner JG, Ling L, Parrish JL, Hughes LF, Caspary DM. Age-related changes in glycine receptor subunit composition and binding in dorsal cochlear nucleus. Neuroscience. 2009;160(1):227-39. [PMID:19217931] http://dx.doi.org/10.1016/j.neuroscience.2009.01.079

38. National Institute on Deafness and other Communication Disorders. Quick statistics [Internet]; 2010 [cited 24 Aug 2013]. Available from: http://www.nided.nih.gov/health/ statistics/Pages/quick.aspx

39. Dille MF, McMillan GP, Reavis KM, Jacobs PG, Fausti SA, Konrad-Martin D. Pre-treatment ototoxicity risk assessment for Veterans prescribed cisplatin. Association for VA Hematology/Oncology; 2012 Oct 19-21; Omaha, NE.

40. McMillan GP, Reavis KM, Konrad-Martin D, Dille MF. The statistical basis for serial monitoring in audiology. Ear Hear. 2013;34(5):610-18. [PMID:23563060] http://dx.doi.org/10.1097/AUD.0b013e31828a21b3

41. Konrad-Martin D, Jacobs PG, McMillan GP, Reavis KM, Dille MF. Usefulness of source knowledge for detecting ototoxicity using DPOAEs. Midwinter Meeting of the Association for Research in Otolaryngology; 2012 Feb; San Diego, CA.

42. Reavis K, McMillan G, Konrad-Martin D, Jacobs P, Dille MF. Dose-related effect of cisplatin on distortion product otoacoustic emissions in Veterans. Midwinter Meeting of the Association for Research in Otolaryngology; 2012 Feb; San Diego, CA.

43. Henry JA, Jastreboff MM, Jastreboff PJ, Schechter MA, Fausti SA. Guide to conducting tinnitus retraining therapy initial and follow-up interviews. J Rehabil Res Dev. 2003;40(2):157-77. [PMID:15077641]

http://dx.doi.org/10.1682/JRRD.2003.03.0159

44. Gladd DK, Saunders GH. Ambient noise levels in the chemotherapy clinic. Noise Health. 2011;13(55):444-51. [PMID:22122961] http://dx.doi.org/10.4103/1463-1741.90322

45. Gordon JS, Phillips DS, Helt WJ, Konrad-Martin D, Fausti SA. Evaluation of insert earphones for high-frequency bedside ototoxicity monitoring. J Rehabil Res Dev. 2005; 42(3):353-61. [PMID:16187247] http://dx.doi.org/10.1682/JRRD.2004.06.0093 
JRRD, Volume 51, Number 1, 2014

46. American National Standards Institute. ANSI S3-1-1996. In: American National Standards Specification for Audiometers. New York (NY): ANSI; 1999.

47. Lee J, Kim J. The maximum permissible ambient noise and frequency-specific averaging time on the measurement of distortion product otoacoustic emissions. Audiology. 1999;38(1):19-23. [PMID:10052832]

http://dx.doi.org/10.3109/00206099909072998

Submitted for publication April 17, 2013. Accepted July 9, 2013.
This article and any supplementary material should be cited as follows:

Konrad-Martin D, Reavis KM, McMillan G, Helt WJ, Dille M. Proposed comprehensive ototoxicity monitoring program for VA healthcare (COMP-VA). J Rehabil Res Dev. 2014;51(1):81-100.

http://dx.doi.org/10.1682/JRRD.2013.04.0092

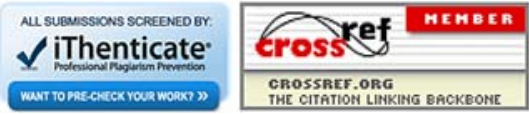

